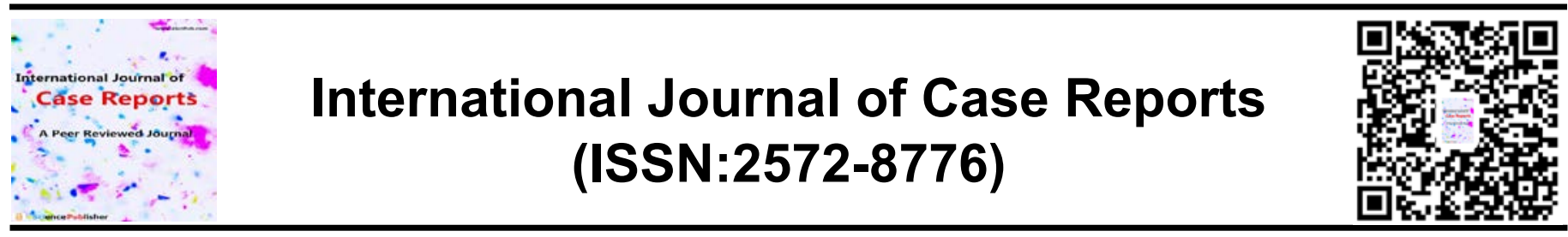

\title{
Immunotherapy induced pyrexia and the role of PET/CT
}

\author{
Luke McLean, Jennifer A Soon, Andrew Haydon \\ Dept. Medical Oncology, The Alfred Hospital, Victoria, Melbourne, Australia
}

\begin{abstract}
Background: Immunotherapy has revolutionised the management of metastatic melanoma, however, immune-related adverse

${ }^{*}$ Correspondence to Author: events remain an important complication of therapy. We hypothesise pyrexia is a rare presentation that may herald the develAndrew Haydon opment of immune-related toxicities and identify a potential role for fluorodeoxyglucose-positron emission tomography (FDG PET) in the earlier diagnosis of these toxicities.

Case Presentation: We report a case of a 54-year-old man with metastatic melanoma, on combination immunotherapy with ipilumimab and nivolumab, where several days of fever heralded the development of clinical enterocolitis. He ultimately required treatment with infliximab with quick resolution of his symptoms. FDGPET imaging performed as a work up for his pyrexia demonstratThe Alfred Hospital, 55 Commercial Rd, Melbourne VIC 3004 ed extensive entero-proctocolitis and a follow up FDG-PET 3 How to cite this article:

Luke McLean, Jennifer A Soon, Andrew Haydon. Immunotherapy induced pyrexia and the role of PET/ CT . International Journal of Case months post infliximab demonstrated complete resolution of the entero-proctocolitis and ongoing complete extracranial response of the melanoma.

Conclusion: Early FDG-PET in patients with unexplained pyrexia on immunotherapy may help in an earlier diagnosis of immune-related toxicities allowing prompt initiation of therapy and a reduction in morbidity and mortality associated with these treat- Website: http://escipub.com/ ments.
\end{abstract}

Keywords: Immunotherapy, metastatic melanoma, immune-related adverse events, pyrexia, colitis, FDG-PET 


\section{BACKGROUND}

According to the Australian Institute of Health and Welfare there were an estimated 13,941 new cases of melanoma diagnosed in Australia in 2017 representing $10.4 \%$ of all new cancer diagnoses (1). Historically, metastatic melanoma has been known for its aggressive course and treatment-resistant behaviour. However by targeting antitumor immune responses, immune checkpoint inhibitors have revolutionised the management of metastatic disease and have demonstrated clinical responses not only in metastatic melanoma but in numerous other malignancies. These powerful therapies include ipilumimab, an anticytotoxic $T$ lymphocyte associated antigen 4 antibody, as well as the anti-programmed death-1 antibodies nivolumab and pembrolizumab.

Despite the significant improvements in overall survival with the use of immunotherapy for the management of metastatic melanoma, immunerelated adverse events remain an important complication of therapy especially in those receiving dual or combination immunotherapy (2) (3). In the following case report we hypothesise that pyrexia is a rare presentation that may precede severe immune-related toxicities such as hepatitis or colitis. We present a case where several days of fever heralded the development of clinical enterocolitis and review the potential role for early fluorodeoxyglucose-positron emission tomography (FDG -PET) in the work up of unexplained pyrexia in patients receiving immunotherapy, especially combination therapy.

\section{CASE PRESENTATION}

The following case concerns a 54-year-old man with metastatic melanoma (BRAF wild type, NRAS mutant) diagnosed in September of 2016 with intracranial and mediastinal sites of metastatic disease. He had previously received 19 cycles of single agent nivolumab without any grade 2 or higher toxicities. However, in the setting of increasing focal seizures despite antiepileptic therapy and previous treatment with stereotactic radiotherapy, magnetic resonance imaging was performed demonstrating progression of a right posterior frontal lesion and he was subsequently commenced on combination ipilumimab $(3 \mathrm{mg} / \mathrm{kg})$ and nivolumab $(1 \mathrm{mg} / \mathrm{kg})$.

After 2 cycles of combination therapy he was admitted with pyrexia, rigors and mild epigastric pain. He did not have any diarrhoea and was commenced empirically on intravenous piperacillin/tazobactam therapy. A computerised axial tomography (CT) scan was performed to investigate his fevers and was unremarkable; of note his bowel appeared normal. A diagnostic workup performed under the guidance of the Infectious Disease team was also unremarkable.

Over the following 4 days febrile episodes up to $39.3^{\circ} \mathrm{C}$ were recorded daily despite broadspectrum antibiotics. Intravenous antibiotics were ceased after 48 hours and the cause of his fevers remained unknown. On day 5 his alanine transaminase (ALT) rose to 180 (normal < 40) consistent with grade II hepatitis and prednisolone $1 \mathrm{mg} / \mathrm{kg}$ was commenced. At this stage an FDG-PET scan was ordered to further investigate his unexplained pyrexia and to exclude progression of melanoma as a cause of his fevers. As seen below the PET scan demonstrated extensive enteroproctocolitis (Image 1A). On day 7 he developed diarrhoea and abdominal discomfort with 8-10 loose bowel motions per day; some with bright red blood. An infective colitis was ruled out and on day 8 he received intravenous methylprednisolone $250 \mathrm{mg}$ daily for 3 days. By day 10 , diarrhoea had increased to 16 times per day consistent with grade 3 colitis. He was treated with infliximab $5 \mathrm{mg} / \mathrm{kg}$, and his condition responded quickly. He was prescribed a weaning regimen of prednisolone with resolution of symptoms over 4 days. A follow up $\mathrm{PET} / \mathrm{CT} 3$ months later demonstrated complete resolution of the enterocolitis (Image 1B) as 
well as ongoing extracranial metabolic remission of his melanoma.

We hypothesise that in this case the earlier use of FDG-PET may have allowed for an earlier diagnosis of colitis resulting in more timely institution of steroids and infliximab.

\section{DISCUSSION}

The use of immunotherapy in the setting of stage IV melanoma has become standard of care in Australia. The use of ipilumimab and nivolumab/pembrolizumab have all demonstrated clinical benefit in stage IV disease and are now available via the Australian Pharmaceutical Benefits Scheme. The CheckMate 067 trial analysed 945 patients with previously unresectable stage III or IV melanoma randomising them to receive nivolumab and ipilumimab or either agent alone with treatment being continued until disease progression or unacceptable toxicity. The overall survival rate at 3 years was $58 \%$ in the combination group, $52 \%$ in the nivolumab group and $34 \%$ in the ipilumimab group (4). Treatment-related adverse effects were significantly higher with combination therapy, with grade $3 / 4$ adverse events occurring in 59\% of patients in the combination group, $21 \%$ on single agent nivolumab and $28 \%$ on ipilumimab alone (4). As a result of this trial a number of
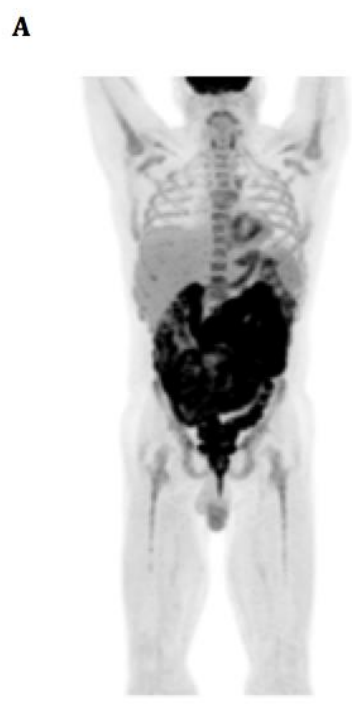

patients are now being commenced on combination immunotherapy in the first line setting. The significant risk of immune-related adverse events with this treatment highlights the need to be able to effectively diagnose these toxicities and institute early immunosuppression to reduce the morbidity associated with these therapies.

The Product Information for nivolumab, pembrolizumab and ipilumimab all list pyrexia as a common toxicity and in the CheckMate 067 trial $5.8 \%$ of patients on nivolumab developed fever, $18.5 \%$ in the combination group and $6.8 \%$ in the ipilimumab group. There is, however, nothing in the literature looking at the association between pyrexia and the subsequent development of immune-related adverse events.

As suggested in this case, we hypothesize that the fever was the first clue to this patient's impending severe immune-related toxicity. Our patient subsequently developed grade II hepatitis as well as grade III colitis. We theorize that if a FDG-PET scan rather than CT was performed, features of colitis would have been more-readily identified and would have led to an earlier institution of steroids, shortened duration of symptoms and potentially eliminated the need for infliximab.

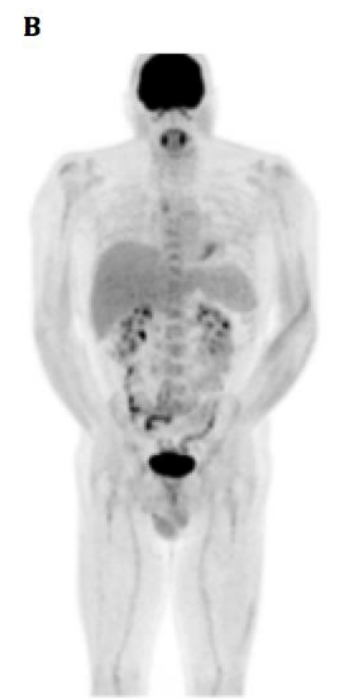

Figure 1: PET/CT scan showing extensive enterocolitis prior to infliximab (A) (September 2017) and 3 months post infliximab (B), showing complete resolution of enterocolitis and ongoing complete response of the melanoma (December 2017.) 
FDG PET-CT has been adopted to assist in the initial staging and in evaluating a response to treatment in patients with metastatic melanoma. However its role may extend further in the era of immunotherapy as there is also now some literature, in the form of case series and reports, looking at patients receiving ipilumimab and the detection of various immune-mediated side effects with FDG PET-CT after treatment including: hypophysitis (5), colitis (6), hepatitis (7) and adrenalitis (8). A case series in Academic Radiology in 2017 also presented 4 cases, including autoimmune pancreatitis, colitis, hypophysitis and thyroiditis, all of which were identified incidentally on routine PET-CT post treatment with ipilumimab allowing an early initiation of appropriate therapy (9). A similar case was also presented in the Clinical Nuclear Medicine Journal where a restaging FDG PET-CT post 3 cycles of pembrolizumab (on a background of previous ipilumimab therapy) demonstrated diffuse increased uptake throughout the body of the pancreas suggestive of pembrolizumab-induced pancreatitis (10). In that case the patient was asymptomatic but a diagnosis was confirmed with raised amylase and lipase levels allowing prompt initiation of steroid therapy.

\section{CONCLUSION}

Immune-related adverse events are a wellrecognised toxicity in the era of immunotherapy. Pyrexia may be an early feature of these toxicities and FDG PET-CT may offer a potential way of identifying these events in their earlier stages allowing prompt initiation of treatment. In this case had we performed a FDG PET-CT earlier as a part of his work-up then this may have allowed for an earlier diagnosis, earlier initiation of steroid therapy, possible avoidance of infliximab and a subsequent reduction in morbidity.

\section{Acknowledgements}

Consent for Publication: Written consent obtained.
Competing Interests/Conflict of interest: the authors declare that they have no competing interests

Funding: no funding was received for this study

Authors' contributions: LM, JS and AH all contributed to the authorship of this manuscript. All authors read and approved the final manuscript.

\section{BIBLIOGRAPHY}

1. Australian Institute of Health and Welfare. Cancer in Australia 2017. Cancer series no 101. 2017; Cat. no. CAN 100.

2. Larkin J, Chiarion-Sileni V, Gonzalez R, Grob JJ, Cowey CL, Lao CD, et al. Combined Nivolumab and Ipilimumab or Monotherapy in Untreated Melanoma. New England Journal of Medicine. 2015;373(1):23-34.

3. Robert C, Schachter J, Long GV, Arance A, Grob JJ, Mortier L, et al. Pembrolizumab versus Ipilimumab in Advanced Melanoma. New England Journal of Medicine. 2015;372(26):2521-32.

4. Wolchok JD, Chiarion-Sileni V, Gonzalez R, Rutkowski P, Grob J-J, Cowey CL, et al. Overall Survival with Combined Nivolumab and Ipilimumab in Advanced Melanoma. New England Journal of Medicine. 2017;377(14):1345-56.

5. van der Hiel B, Blank CU, Haanen JB, Stokkel MP. Detection of early onset of hypophysitis by (18)F-FDG PET-CT in a patient with advanced stage melanoma treated with ipilimumab. Clin Nucl Med. 2013;38(4):e182-4.

6. Lyall A, Vargas HA, Carvajal RD, Ulaner G. Ipilimumab-induced colitis on FDG PET/CT. Clin Nucl Med. 2012;37(6):629-30.

7. Raad RA, Pavlick A, Kannan R, Friedman KP. Ipilimumab-induced hepatitis on 18F-FDG $\mathrm{PET} / \mathrm{CT}$ in a patient with malignant melanoma. Clin Nucl Med. 2015;40(3):258-9.

8. Bacanovic S, Burger IA, Stolzmann P, Hafner J, Huellner MW. Ipilimumab-Induced Adrenalitis: A Possible Pitfall in 18F-FDG-PET/CT. Clin Nucl Med. 2015;40(11):e518-9.

9. Wachsmann JW, Ganti R, Peng F. Immunemediated Disease in Ipilimumab Immunotherapy of Melanoma with FDG PET-CT. Acad Radiol. 2017;24(1):111-5.

10. Alabed YZ, Aghayev A, Sakellis C, Van den Abbeele AD. Pancreatitis Secondary to AntiProgrammed Death Receptor 1 Immunotherapy Diagnosed by FDG PET/CT. Clin Nucl Med. 2015;40(11):e528-9. 